\title{
Trayectoria Académica de Estudiantes Universitarios
}

\section{Academic Career of University Students}

\begin{abstract}
Fabián Eugenio Bravo Guerrero ${ }^{1}$, Lourdes Illescas-Peña ${ }^{2}$, Mario Patricio Peña Ortega ${ }^{3}$
INFORMACIÓN DEL ARTÍCULO

Fecha de recepción: 29 de Enero de 2020.

Fecha de aceptación: 2 de Junio de 2020

\footnotetext{
1 Magíster en Educación de la Universidad Tecnológica América. Docente e Investigador de la Docente e Investigador de la
Universidad de Cuenca-Ecuador. E-mail: fabian.bravo@ucuenca.edu.ec Código ORCID:

http://orcid.org/0000-0002-0372-2071.
}

${ }^{2}$ Magister en Análisis y Visualización de Datos Masivos de la UNIR. Docente e Investigadora de la Universidad de

\section{Resumen}

Si bien las carreras cuentan con una planificación de currículo, en la práctica el estudiante demora algún tiempo adicional en cumplir su programa. En algunas carreras esa demora puede ser excesiva, implicando retrasos y la pérdida de recursos para el estudiante y la institución. Esta investigación se realizó con una cohorte que inició en marzo del 2013 y culminó en julio del 2018, con el objetivo de analizar longitudinalmente el progreso académico de los estudiantes, y evidenciar el desfase entre el avance ideal y el avance real en cada período, y así poder definir políticas de optimización de la gestión educativa. Se encontró una facultad donde sus estudiantes demoraron un 46,6\% más que el plazo ideal; adicionalmente, aquellos estudiantes, cuyos padres son profesionales o que desempeñan cargos altos, tiene avances académicos estadísticamente superiores a otros.
\end{abstract} Cuenca-Ecuador

E-mail: lourdes.illescasp@ucuenca.edu.ec Código ORCID:

http://orcid.org/0000-0002-0027-439X.

${ }_{3}^{3}$ Magíster en Gestión de Proyectos de la Universidad de las Fuerzas Armadas. Docente e Investigador de la Universidad de Cuenca-Ecuador.

E-mail: mario.penao@ucuenca.edu.ec Código ORCID:

http://orcid.org/0000-0002-3986-7707.

El artículo es resultado del proyecto de Investigación: "Relación entre el tiempo de aprobación de un plan de estudios y la condición socioeconómica del estudiante en la Universidad de Cuenca", ganador del XIV Concurso de Proyectos de Investigación, financiado por la Dirección

CITACIÓN: Bravo Guerrero, F.E., Illescas-Peña, L., \& Peña Ortega, M.P. (2020). Trayectoria Académica de Estudiantes Universitarios. Podium, 37, 27-42. doi:10.31095/podium.2020.37.3

\section{ENLACE DOI:}

http://dx.doi.org/10.31095/podium.202 0.37 .3

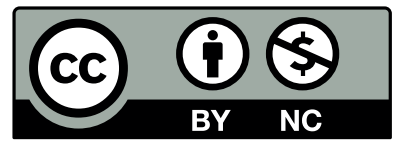

\section{Palabras Clave:}

Avance académico, duración de estudios, variables socioeconómicas, educación superior, estudiante universitario, Abandono.

\section{Clasificación JEL: I21.}

\begin{abstract}
Although the careers have a curriculum planning, in practice the student takes some additional time to complete his program. In some careers, this delay can be excessive, implying delays and the loss of resources for the student and the institution. This research was carried out with a cohort that began in March 2013 and ended in July 2018, with the aim of longitudinally analyzing the academic progress of the students, and evidencing the gap between the ideal progress and the real progress in each period, and Thus, to define optimization policies for educational management. A faculty was found where its students took $46.6 \%$ more than the ideal term; Additionally, those students, whose parents are professionals or who hold high positions, have academic progress that is statistically superior to others.
\end{abstract}

\section{Keywords:}

Academic progress, duration of studies, socioeconomic variables, higher education, university student, Dropout.

JEL Classification: I21. 


\section{Introducción}

Los estudiantes universitarios enfrentan ciertas dificultades que pueden retrasar la culminación de sus estudios: bajo rendimiento debido al bachillerato de procedencia (Vargas, Ramírez, Cortés, Farfán y Heinze, 2011), problemas de integración social e institucional (Tinto, 1989), deserción por trabajo (Belvis, Moreno y Ferrer, 2009) o necesidades económicas (Soria-Barreto y ZúñigaJara, 2014; Pineda-Báez, Pedraza- Ortiz y Moreno, 2011). Dichas dificultades pueden ocurrir en diferentes momentos de sus estudios, especialmente al ingreso $\mathrm{y}$ al egreso de la carrera (Zandomeni y Canale, 2010).

Históricamente existen carreras universitarias que tienen un alto rigor $y$ dificultad, tanto en las áreas técnicas, como de la salud, así como en algunas asignaturas puntuales, esto ocasiona que en algún momento los estudiantes reprueben asignaturas y vayan demorando la culminación de su plan de estudios en el plazo ideal, y retrasen su incorporación a la vida profesional. Las instituciones educativas, por su parte, deben planificar cursos extras, disponer de un mayor número de docentes, $\mathrm{y}$ espacio físico para abastecer la demanda de matrícula de los alumnos que repiten asignaturas, y en las universidad públicas los recursos son escasos y restringidos (Bravo, Illescas, Larriva y Peña, 2017; Giovagnoli, 2001).

La pregunta que orientó la investigación buscaba describir y cuantificar las trayectorias académicas de los estudiantes universitarios, para así determinar ¿En qué tiempo se aprueban los programas de estudios en las carreras de la Universidad de Cuenca? y ¿Qué relación tienen los avances en las carreras con las características socio-económicas de los estudiantes de la Universidad de Cuenca? Zandomeni y Canale (2010) indican que se requiere una cuantificación en la búsqueda de una primera aproximación al objeto de estudio con propósitos descriptivos, para luego avanzar en la identificación de los posibles factores que configuran dichas características.

La investigación consistió en el seguimiento de las trayectorias académicas de los estudiantes pertenecientes a 44 carreras de la Universidad de Cuenca, quienes ingresaron al primer semestre en marzo del 2013, hasta julio del 2018. Se analizan los avances que logran los estudiantes al cursar sus carreras, se los compara con los plazos ideales establecidos en sus planes curriculares, y se calcula el tiempo que les tomaría completarlas. También se estudió la relación que tienen los avances académicos con factores socioeconómicos. Dar un seguimiento detallado a la trayectoria académica del estudiante, puede ayudar a cuantificar esas demoras e identificar con detalle algunos problemas que puedan existir y ser fuente de información para la planeación y mejora de la calidad educativa (Zandomeni y Canale, 2010), estas demoras pueden significar pérdidas de tiempo y recursos para los estudiantes y la institución educativa (Bravo et al., 2017). 


\section{Revisión de literatura}

Algunos autores encuentran similitud en los términos: trayectorias académicas, trayectorias educativas y trayectorias escolares, aunque prefieren el primero porque consideran que hay mayor afinidad con la educación formal, y en particular con la educación superior, el segundo término es demasiado general, mientras que el tercero se refiere a la escuela primaria (Zandomeni y Canale, 2010). Para Sepúlveda (2013), la trayectoria académica es el resultado del recorrido curricular que realiza un estudiante teniendo en cuenta el tiempo de duración de la carrera, la regularidad en los estudios y el egreso. Dentro de estas definiciones, se distinguen dos tipos de trayectoria, aquella planificada en el currículo de la carrera y que es la ideal, y una segunda trayectoria que es la que realmente el estudiante completa. Terigi (2007) manifiesta que las trayectorias teóricas expresan itinerarios en el sistema que siguen la progresión lineal prevista por éste en los tiempos marcados por una periodización estándar. Por su parte, Zandomeni y Canale (2010) indican que las trayectorias reales muestran las formas o los modos en que transitan los estudiantes por la institución educativa. Para esta investigación, la trayectoria académica ideal está dada por los créditos que han sido planificados para cada período académico en cada carrera, esto consta en el plan curricular; mientras, la trayectoria académica real muestra el ritmo al que los estudiantes aprueban los créditos en su permanencia académica.

Para Doray, Picard, Trottier y Groleau
(2009) muchos estudiantes toman trayectorias académicas que ellos las denominan atípicas, que se caracterizan por retiros frecuentes, reingresos, o cuando deciden por cambiarse a segundas carreras; esos estudiantes no toman los niveles esperados de la progresión académica. Camarena, Chávez y Gómez (1985), asocian a la trayectoria o recorrido escolar con otros conceptos ligados a la eficiencia terminal y la deserción escolar. La eficiencia terminal se refiere al porcentaje de estudiantes graduados en relación con el número de los que ingresaron (Piña y Pontón, 1997); y, la deserción escolar "se define como la acción de abandonar la universidad por cualquier causa" (Fernández y Silva, 2014, p. 36), aunque Tinto (1982) considera que hay deserción si no hay actividad durante tres semestres consecutivos.

En la misma línea, el rendimiento académico es considerado como la medida del conocimiento logrado en un tema o una asignatura (Lamas, 2015), los docentes realizan la evaluación del desempeño de los estudiantes y del logro de los aprendizajes, y lo plasman en una calificación (Morales, Morales, y Holguín, 2016), ya sea esta cuantitativa o cualitativa, es el reflejo de lo que el estudiante demuestra o logra hacer con lo que ha aprendido (Lerner, 2011). Pero los estudiantes que quieren lograr aprendizajes duraderos, tratan de comprender los temas, sus aplicaciones y relacionarlos entre sí (Fenollar, Cuestas, y Román, 2008), mientras, otros sólo estudian para las evaluaciones y logran aprendizajes superficiales y poco duraderos (Kohler, 2013). 
En el rendimiento académico intervienen múltiples factores, que pueden ser personales como el nivel intelectual, la personalidad, la motivación, las aptitudes, los intereses, los hábitos de estudio, la autoestima (Lamas, 2015), otros relacionados con el entorno familiar y social (De Garay Sánchez, 2004), como la violencia, seguridad o trabajo (Garzón, Rojas, Riesgo, Pinzón, y Salamanca, 2010); también hay factores relacionados con el docente, como la relación con el alumno, la calidad del maestro, el ambiente que se genera en la clase (Parra et al., 2013); o factores académicos como los objetivos, contenidos, metodología, recursos didácticos, sistema de evaluación, infraestructura (Morales, Morales, y Holguín, 2016).

Para medir la trayectoria académica en el Ecuador, es necesario basarse en el marco legal de educación superior que regula el número de horas, el número máximo de asignaturas y la duración de las carreras, para el período investigado, en el art. 17 del Reglamento de Régimen Académico (Consejo de Educación Superior, 2017) determinaba que las carreras de grado podían tener entre 7200 horas para licenciaturas, 8000 horas para las ingenierías, arquitectura, odontología y veterinaria, y 13160 horas para medicina entre la formación y el internado. El art. 357 de la Constitución del Ecuador (Asamblea Constituyente, 2008) indica que el estado garantiza el financiamiento a las instituciones de educación superior, pero la distribución de estos recursos estará basada en la calidad de la educación, por eso las instituciones deben esforzarse por obtener una buena calificación en el proceso de acreditación de las carreras que se realizan periódicamente y en la cual se consideran las tasas de retención y aprobación estudiantil como elementos de evaluación.

En la Universidad de Cuenca, el sistema de mallas curriculares por créditos permite determinar el número mínimo de créditos que un alumno necesita para completar una carrera. Para ello, es necesario considerar que en cada semestre el estudiante registra un avance en función del número de créditos que ha aprobado, siendo la nota mínima de aprobación de $60 \%$ del aprovechamiento. En este proyecto, esa información es presentada de modo gráfico, facilitando la visualización en una línea de tiempo, con el propósito de identificar cualquier anomalía en la trayectoria de cada estudiante o de los estudiantes en cada carrera. También, se ha realizado un cálculo que estima la duración de los estudios en cada una de las carreras de la Universidad de Cuenca. Estos elementos permiten el análisis de las trayectorias académicas en una carrera específica y cohorte de estudios determinada.

Las investigaciones que han trabajado sobre trayectorias académicas se complementan con temas de deserción, retención estudiantil y eficiencia terminal. Algunas investigaciones desarrollaron sus propias metodologías para analizar la trayectoria académica, mientras, otras tratan de encontrar causalidad entre algunas variables asociadas al estudiante y la trayectoria 
académica. La mayoría analizan algunos indicadores de trayectoria de forma cuantitativa (Zandomeni y Canale, 2010), otras lo hacen de forma gráfica para poder visualizar cómo se desarrolla el avance con el paso del tiempo (Peña, Bravo, e Illescas-Peña, 2019), aunque, en otras aplican técnicas cualitativas para facilitar la comprensión de las trayectorias académicas de los estudiantes (Sepúlveda, 2013). Solano Lucas, Frutos y Cárceles Breis (2004) estudiaron la trayectoria académica mediante el análisis longitudinal de los recorridos de los estudiantes en las carreras que cursan para determinar los tiempos que demoran en sus estudios, e identificar aquellas carreras que toman más y menos tiempo en ser cursadas. García-Castro y Bartolucci (2007) encontraron que algunos factores de la vida estudiantil previa, tales como la edad, género, trayectoria escolar, ingresos familiares, escolaridad de los padres, pueden estar relacionados con el éxito o fracaso del estudiante en su posterior trayectoria académica universitaria. Martínez-Padilla y PérezGonzález (2008) desarrollaron una metodología que les permite predecir el éxito o fracaso académico, logran una alta capacidad predictiva mediante un índice conformado por 7 variables.

Rembado, Ramírez, Viera, Ros y Wainmaier (2009) hicieron una investigación cualitativa, ahí, los estudiantes encuentran como condicionantes de su trayectoria académica al proceso de enseñanza en la universidad y las dificultades relacionadas con su formación media. Mares et al. (2012) analizaron el rendimiento académico y la deserción, encontraron que el estudiante forja su trayectoria de acuerdo a sus competencias académicas y a la motivación que demuestra por su preparación. Sepúlveda (2013) estudió la diversidad de los estudiantes y sus trayectorias, y encontró que los estudiantes escogen carreras buscando mejorar su posición laboral; destacan factores como el acompañamiento de la familia, perseverancia, autodisciplina y renuncia al descanso. Las trayectorias no sólo tienen que ver con los tiempos que demoran en realizar sus estudios, sino con otras variables como el rendimiento, las tasas de aprobación y reprobación, la deserción y la eficiencia terminal que son importantes para las instituciones educativas de educación superior, porque se pueden tomar decisiones académicas que lleven a la universidad a mejorar en sus índices de eficiencia académica (Bravo et al., 2017).

\section{Metodología}

Esta investigación tiene un enfoque cuantitativo, de tipo descriptivo y correlacional, basada en un estudio longitudinal de cohorte (Labaree, 2013). Involucró a 1900 estudiantes pertenecientes a las 12 facultades de la Universidad de Cuenca, que totalizan 44 carreras. Los estudiantes que fueron investigados ingresaron a sus respectivas carreras en marzo del 2013 y se dio seguimiento a sus avances académicos reales hasta julio del 2018, para luego compararlos con los avances ideales que están descritos en el programa curricular de cada carrera. La Tabla 1 muestra la distribución de los 
Tabla 1 .

Salida semestral de ministros, viceministros y subsecretarios de Estado

\begin{tabular}{|c|c|c|}
\hline FACULTAD & CARRERA & ESTUDIANTES \\
\hline & Administración de Empresas & 100 \\
\hline & Contabilidad y Auditoría & 236 \\
\hline Ciencias & Economía & 45 \\
\hline Económicas y & Ingeniería de Empresas & 21 \\
\hline \multirow[t]{5}{*}{ Administrativas } & Marketing & 24 \\
\hline & Sociología & 14 \\
\hline & Total & 440 \\
\hline & Civil & 69 \\
\hline & Sistemas & 21 \\
\hline \multirow{5}{*}{ Ingeniería } & Eléctrica & 14 \\
\hline & Electrónica y Telecomunicaciones & 35 \\
\hline & Total & 139 \\
\hline & Filosofía, Sociología y Economía & 1 \\
\hline & Lengua y Literatura Inglesa & 11 \\
\hline \multirow{6}{*}{$\begin{array}{l}\text { Filosofía Letras } \\
\text { y Ciencias de } \\
\text { la Educación }\end{array}$} & Periodismo y Comunicación Digital & 24 \\
\hline & Comunicación Organizacional y Relaciones & 30 \\
\hline & Públicas & \\
\hline & Matemáticas y Física & 9 \\
\hline & Educación General Básica & 27 \\
\hline & Total & 102 \\
\hline Agronomía y & Agronomía & 26 \\
\hline \multirow{4}{*}{ Veterinaria } & Veterinaria y Zootecnia & 33 \\
\hline & Total & 59 \\
\hline & Psicología Clínica & 60 \\
\hline & Psicología Educativa & 78 \\
\hline \multirow[t]{5}{*}{ Psicología } & Psicología Social & 22 \\
\hline & Total & 160 \\
\hline & Medicina & 196 \\
\hline & Enfermería & 108 \\
\hline & Terapia Física & 20 \\
\hline Ciencias & Nutrición & 21 \\
\hline \multirow{4}{*}{ Químicas } & Estimulación Temprana & 27 \\
\hline & Laboratorio Clínico & 33 \\
\hline & Imagenología & 26 \\
\hline & Total & 431 \\
\hline
\end{tabular}




\begin{tabular}{llr}
\hline \multirow{2}{*}{ FACULTAD } & \multicolumn{1}{c}{ CARRERA } & ESTUDIANTES \\
\hline \multirow{3}{*}{ Hospitalidad } & Gastronomía & 33 \\
& Hotelería & 31 \\
& Turismo & 52 \\
& Total & $\mathbf{1 1 6}$ \\
\hline \multirow{2}{*}{ Jurisprudencia } & Derecho & 90 \\
& Orientación Familiar & 31 \\
& Trabajo Social & 30 \\
& Total & $\mathbf{1 5 1}$ \\
\hline \multirow{2}{*}{ Arquitectura } & Arquitectura & 63 \\
& Total & $\mathbf{6 3}$ \\
\hline \multirow{2}{*}{ Odontología } & Odontología & 63 \\
& Total & $\mathbf{6 3}$ \\
\hline Artes & Artes Musicales & 16 \\
& Danza y Teatro & 3 \\
& Artes Visuales & 19 \\
& Diseño & $\mathbf{1 9 0 0}$ \\
& Total & 19 \\
\hline
\end{tabular}

Fuente: Elaboración propia.

estudiantes matriculados por carrera $\mathrm{y}$ facultad.

Para graficar las trayectorias académicas de cada estudiante, se requirió determinar los créditos que iba aprobando en cada semestre, y así evidenciar el avance individual por período, logrando al final que consten las trayectorias de todos los estudiantes de una carrera según la metodología propuesta por Illescas-Peña, Peña, Bravo, y Larriva (2018). En la Figura 1 se muestra un ejemplo genérico del gráfico que se elaboró para cada una de las 44 carreras; allí, cada línea representa el avance de un estudiante, algunos culminan su carrera dentro del plazo ideal, otros se atrasan; mientras, algunos desertan.

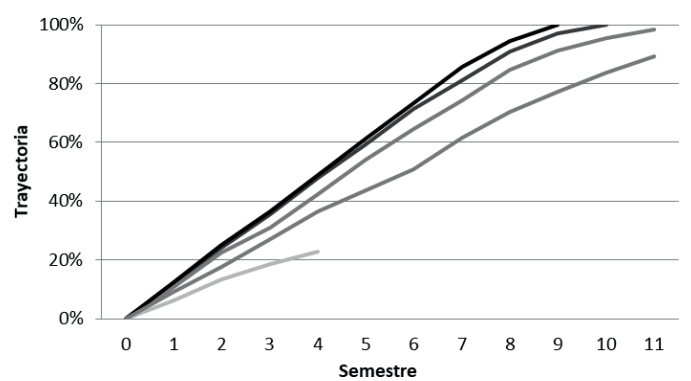

Figura 1. Ejemplo del gráfico de trayectoria académica de los estudiantes de una carrera.

Fuente: Elaboración: Propia.

Para realizar el seguimiento a los avances académicos, se utilizaron los registros académicos, y para analizar las variables socio-económicas de los estudiantes se usaron las bases de datos digitales donde 
se almacena toda esta información, las cuales son gestionadas por el Departamento de Tecnologías de la Información y Comunicación de la Universidad de Cuenca (DTICs). Se obtuvieron las autorizaciones respectivas justificando el uso que se pretendía y manteniendo la confidencialidad en el uso de los datos.

Para determinar cuál es el avance académico de una facultad, se promediaron los créditos que los estudiantes habían aprobado durante el plazo ideal y se lo comparó con el total de créditos que constan en el plan curricular de cada carrera.

Avance Académico $(\%)=\frac{\text { Creditos aprobados promedio }}{\text { Creditos Totales de la Carrera }} \times 100$

Los semestres necesarios para culminar sus estudios quedan definidos por el plan curricular de cada carrera. Sin embargo, dado que en general a los estudiantes les toma más tiempo finalizar los estudios en sus carreras, es necesario estimar los semestres en que los estudiantes de las diferentes facultades culminarían sus estudios, para lo cual se emplea una relación directamente proporcional propuesta por Terigi (2007), que se explica en la Figura 2. $\begin{aligned} & \text { Semestres requeridos para } \\ & \text { culminar los Estudios }\end{aligned}=\frac{\text { Semestres según Plan Curricular }}{\text { Avance Académico en } \%} \times 100$

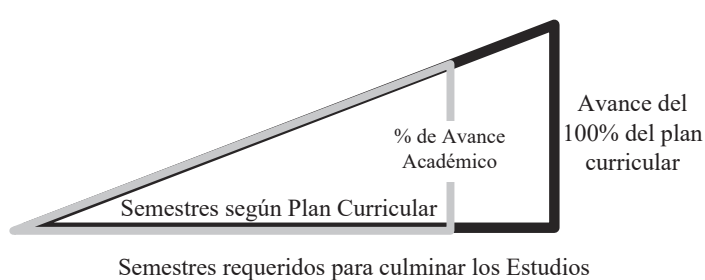

Figura 2. Relación directamente proporcional para el cálculo del número de semestres en que culminarían los estudios los alumnos de las diferentes facultades de la Universidad de Cuenca.

Fuente: Elaboración: Propia.
La relación entre el avance académico y las variables de la ficha socioeconómica se realizó en cuatro carreras seleccionadas por conveniencia, por su alta demanda y prestigio: Medicina, Derecho, Ingeniería Civil y Administración de Empresas. La información de la ficha socio-económica que el proyecto utilizó fue registrada por los estudiantes al inicio de sus estudios en marzo del 2013. Para el análisis de datos se utilizó la herramienta estadística SPSS versión 25.0.

\section{Resultados}

El proyecto de investigación consideró importante la presentación visual de la trayectoria académica para que las autoridades institucionales puedan observar el avance individual de los estudiantes de una carrera, esta información les permitirá tomar acciones o decisiones al detectar problemas académicos e incluso prevenir la deserción de los estudiantes. La Figura 3 presenta el progreso de la trayectoria de cada estudiante durante los semestres de matrícula a partir de marzo del 2013. Se puede observar que pocos estudiantes culminan sus estudios en el plazo ideal de nueve semestres, mientras que, la mayoría logra culminar el plan curricular en uno o dos semestres adicionales al plazo ideal. En el caso de aquellos alumnos que han desertado, su trayectoria se interrumpe. Obtener la información académica de los estudiantes de una carrera, y poder identificar las trayectorias individuales puede permitir a las autoridades académicas identificar dificultades individuales o del grupo, para tomar decisiones que retengan a los 


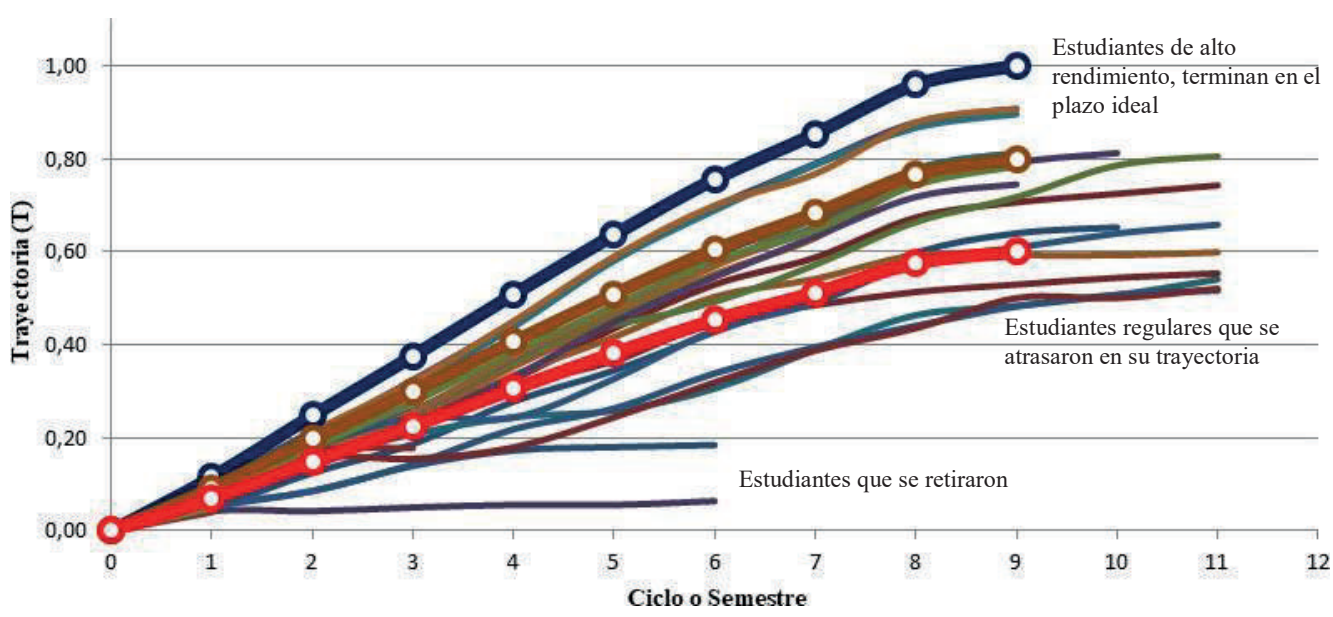

Figura 3. Trayectoria Académica de los estudiantes de la carrera Educación General Básica.

Fuente: Elaboración: Propia.

estudiantes y así evitar su deserción.

Se calculó el avance académico para las 12 facultades y se consideró el porcentaje de créditos que los estudiantes habían logrado aprobar hasta la fecha de culminación de sus estudios, según lo programado en las mallas curriculares. La Figura 4 muestra el resumen de estos avances, considerando que cada avance corresponde a la cohorte que ingresó en marzo del 2013. De los 1900 alumnos investigados, $479(25,21 \%)$ desertaron en diferentes etapas de sus estudios; mientras, 1421 (74,79\%) se mantuvieron estudiando, de ellos han avanzado en promedio un $85,3 \%$ de los créditos correspondientes a sus carreras, con una desviación estándar del 17,9\%. El mínimo avance lo tiene un estudiante que solamente ha aprobado un $9,3 \%$ de los créditos y no se ha retirado; mientras, el máximo avance es del $102,2 \%$ valor que indica que el estudiante ha aprobado más créditos que los que le exige el plan curricular de su carrera.

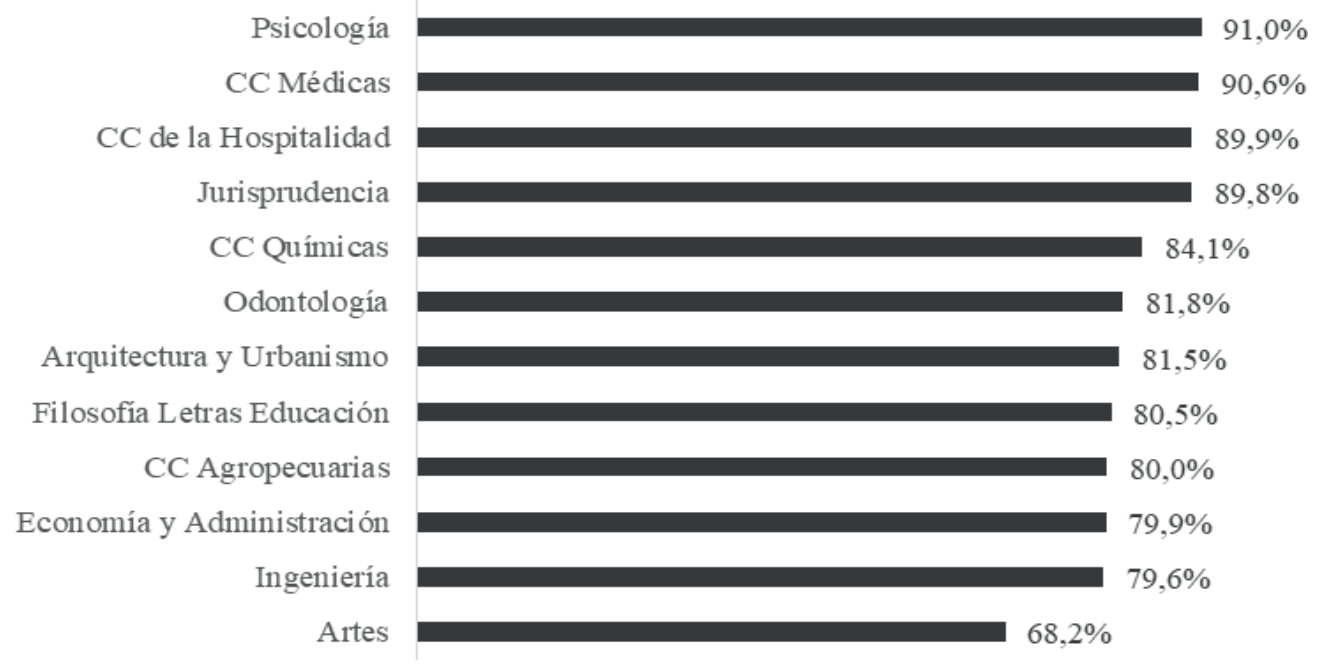

Figura 4. Avance Académico de los estudiantes de las doce facultades de la Universidad de Cuenca. 


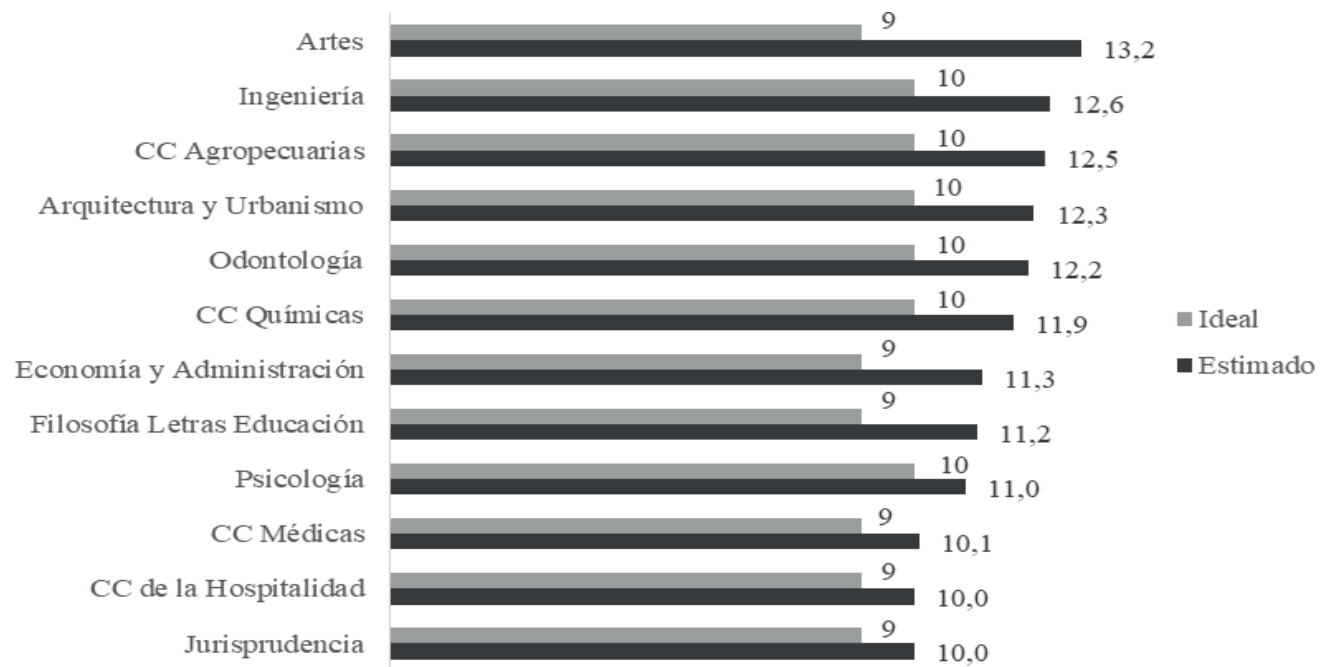

Figura 5. Duración estimada de los estudios en las doce facultades de la Universidad de Cuenca (en semestres).

Una vez calculados los avances de los estudiantes para cada facultad, en relación al número de semestres y créditos necesarios para completar las carreras, se procedió a determinar el número de semestres en que terminarían sus estudios en las diferentes facultades (ver Figura 5). La duración media de los planes de estudios en la Universidad de Cuenca es de 9,57 semestres, y se estima que los alumnos terminarán sus estudios en 11,27 semestres, es decir se demorarían un $17,8 \%$ adicional al plazo ideal, determinado por los planes curriculares.

De forma paralela, dentro del análisis se estableció la relación entre el avance de los estudiantes y los datos socioeconómicos. Para ello, fueron analizados 370 estudiantes pertenecientes a las carreras de: Administración de Empresas, Ingeniería Civil, Derecho y Medicina (Ver Tabla 2).

Dentro de los resultados se determinó que el $91,62 \%$ de los estudiantes son solteros; con respecto al género hay un cierto balance, el $53,24 \%$ son mujeres y el $46,76 \%$ son varones; el $90,81 \%$ viven en zona urbana, el $88,92 \%$ están domiciliados en Cuenca. En relación al colegio de procedencia, 93,78\% pertenecen a la zona urbana; siendo el $54,32 \%$ procedente de colegios particulares. E1 76,22\% ingresó con una edad menor o igual a 18 años. E1 91,62\% lo hizo hasta un año después de haberse graduado del bachillerato. El 81,89\% de los estudiantes no es jefe de familia. Finalmente, $76,49 \%$ proceden de familias con ingresos entre bajos y medios; y, el $24,32 \%$ provienen de familias con responsables del hogar no profesionales, que corresponde a obreros con oficios poco remunerados.

Para determinar si existe una relación entre el avance académico y las variables de la ficha socioeconómica, se determinó normalidad y homocedasticidad tomando en cuenta las categorías de cada una de las variables independientes de la Tabla 2. Aunque algunas variables independientes presentaron igualdad de varianzas, no 
Tabla 2.

Resumen de las variables de la ficha socioeconómica de los estudiantes de las carreras analizadas

\begin{tabular}{|c|c|c|c|}
\hline VARIABLES & ESCALA & $\mathbf{N}$ & \% TOTAL \\
\hline \multirow[t]{4}{*}{ Carrera } & Administración & 51 & $13.78 \%$ \\
\hline & Derecho & 77 & $20.81 \%$ \\
\hline & Ingeniería Civil & 58 & $15.68 \%$ \\
\hline & Medicina & 184 & $49.73 \%$ \\
\hline \multirow{2}{*}{ Es soltero } & No & 31 & $8.38 \%$ \\
\hline & Sí & 339 & $91.62 \%$ \\
\hline \multirow[t]{2}{*}{ Género } & Femenino & 197 & $53.24 \%$ \\
\hline & Masculino & 173 & $46.76 \%$ \\
\hline \multirow[t]{2}{*}{ Zona Colegio } & Rural & 23 & $6.22 \%$ \\
\hline & Urbano & 347 & $93.78 \%$ \\
\hline \multirow[t]{2}{*}{ Tipo Colegio } & Fiscal & 169 & $45.68 \%$ \\
\hline & Particular & 201 & $54.32 \%$ \\
\hline \multirow[t]{2}{*}{ Ingreso hasta 1 año } & No & 31 & $8.38 \%$ \\
\hline & Sí & 339 & $91.62 \%$ \\
\hline \multirow[t]{2}{*}{ Zona Vivienda } & Rural & 34 & $9.19 \%$ \\
\hline & Urbano & 336 & $90.81 \%$ \\
\hline \multirow[t]{4}{*}{ Ingresos } & Bajo & 173 & $46.76 \%$ \\
\hline & Medio & 110 & $29.73 \%$ \\
\hline & Alto & 54 & $14.59 \%$ \\
\hline & Muy alto & 33 & $8.92 \%$ \\
\hline \multirow[t]{2}{*}{ Es jefe de familia } & No & 303 & $81.89 \%$ \\
\hline & Sí & 67 & $18.11 \%$ \\
\hline \multirow[t]{3}{*}{ Profesión reducida } & I (Altos cargos) & 68 & $18.38 \%$ \\
\hline & II (Profesionales) & 212 & $57.30 \%$ \\
\hline & III (No Profesionales) & 90 & $24.32 \%$ \\
\hline \multirow[t]{3}{*}{ Edad de ingreso en } & Menor o igual a 18 años & 282 & $76.22 \%$ \\
\hline & Entre 19 y 21 años & 85 & $22.97 \%$ \\
\hline & Mayor a 21 años & 3 & $0.81 \%$ \\
\hline \multirow{2}{*}{$\begin{array}{l}\text { Domicilio en } \\
\text { Cuenca }\end{array}$} & No & 41 & $11.08 \%$ \\
\hline & Sí & 329 & $88.92 \%$ \\
\hline \multirow{2}{*}{$\begin{array}{l}\text { Ciudad de } \\
\text { procedencia }\end{array}$} & Foráneo & 77 & $20.81 \%$ \\
\hline & Local & 293 & $79.19 \%$ \\
\hline
\end{tabular}

Fuente: Elaboración propia. 
cumplían con el criterio de normalidad. Por lo tanto, se aplicaron pruebas no paramétricas (Prueba de Kruskal- Wallis y Prueba de U de Mann-Whitney) para determinar diferencias entre las medias de avance respecto a cada categoría. En el análisis, se pudo determinar que en las carreras técnicas o administrativas, las medias de los avances son inferiores a las carreras sociales o del área de medicina (p-valor $<0.001)$. Se consideraron 4 poblaciones definidas por cada carrera, sobre las cuales se determinó la influencia de las variables independientes. Para las carreras de Administración y Derecho, no se logró determinar variables socioeconómicas que influyeran en el avance de los estudiantes. En la carrera de Ingeniería Civil, aunque la población de los que no son solteros es pequeña (sólo 6.9\%) se puede afirmar que los estudiantes en esta condición presentan una media inferior $(62.09 \%$ de avance) respecto a los solteros ( $72.53 \%$ de avance).

En la carrera de Medicina se identificó una correlación significativa entre el avance y la profesión del jefe de familia, en la que aquellos estudiantes cuyos padres tienen una profesión y ocupan altos cargos poseen los mayores avances $(p$-valor $=0.036)$. Los estudiantes en los que el jefe de familia tiene una profesión de categoría I (Profesional con altos cargos), presentan un mayor avance (93.54\% de avance) que los de categoría II (89.92\% de avance). Se conformaron 2 grupos que comparten la categoría III (93.45\% de avance), por lo que esta última se solapa tanto con la categoría I como con la categoría II. Para esta carrera, aunque la muestra no es muy representativa, $(3.26 \%$ de los estudiantes ingresaron después de 1 año de haberse graduado) se puede afirmar que los estudiantes que ingresan inmediatamente (hasta 1 años después) luego de haberse graduado tienen en promedio una media superior (97.60\% del avance) a los que ingresan en una condición diferente $(85.40 \%$ del avance $),(p$-valor $=0.004)$.

Finalmente, la edad de ingreso es un factor determinante del avance, pues quienes ingresaron con una edad inferior o igual a 18 años presentan una media de avance $(92.49 \%$ de avance) estadísticamente superior $(p$-valor $=0.026)$ a quienes ingresaron con una edad igual o superior a 19 años ( $75.64 \%$ de avance).

En la Universidad de Cuenca, las facultades que poseen las carreras que menos tiempo toman en ser completadas son: Jurisprudencia, Hospitalidad, Ciencias Médicas y Psicología con un semestre adicional al plazo ideal. En cuanto a las facultades donde sus carreras toman más tiempo en ser completadas son las técnicas: Ingeniería, Ingeniería Agropecuaria y Arquitectura con aproximadamente 2,5 semestres adicionales, mientras la facultad de Artes tiene una demora de 4,2 semestres. Hay una coincidencia parcial con la investigación de Solano Lucas, Frutos y Cárceles Breis (2004), quienes indican que "las titulaciones en las que el alumnado consume menos tiempo para alcanzar el éxito son todas de las ramas más numerosas (Humanidades y Ciencias Sociales). Por lo que respecta a las carreras que más tiempo dedican sus 
alumnos para obtener el título, son las de Ciencias de la Salud y de Ciencias Experimentales" (p.232).

En cuanto a la relación entre la profesión del responsable del hogar y el avance académico del estudiante, se encontró esa asociación en la Facultad de Ciencias Médicas; esta información tiene relación con la investigación de Ishitani y Snider (Citados por Soria-Barreto y Zúñiga-Jara, 2014), quienes indican que los estudiantes provenientes de hogares con padres de mayor nivel educativo y más altos ingresos tienen mayor probabilidad de completar sus estudios; también en un informe para América Latina y el Caribe del Banco Mundial (Ferreyra et al., 2017) se indica que los estudiantes que vienen de familias con niveles de ingresos bajos, tienen un nivel de preparación académica menor que aquellos estudiantes cuyas familias tienen ingresos más altos.

Respecto a la edad de ingreso, en la Universidad de Cuenca, el 76,22\% de los estudiantes lo hizo con 18 años o menos, el 23,78\% con más de 18 años; por otra parte, responden que el 8,38\% ingresó más de un año después de graduarse del colegio. En contraste, en una investigación realizada en la Universidad Juárez Autónoma da Tabasco, en México por Rozo (2008) se determinó que el 23\% ingresó más de un año después de graduados; mientras, el 24\% tiene más de 20 años al ingresar. En la ecuatoriana el $45,68 \%$ de estudiantes viene de una unidad educativa fiscal, mientras en la universidad mexicana el $95 \%$ vienen de una escuela pública, marcándose una fuerte diferencia entre los hallazgos de las dos investigaciones.

\section{Conclusiones}

Las gráficas de trayectoria académica permiten visualizar el avance del estudiante e identificar comportamientos individuales o del grupo; con esta información visual la autoridad puede tomar decisiones académicas que permitan corregir los problemas académicos detectados y mejorar el proceso educativo. Se puede identificar a la Facultad de Artes y a las Facultades del Área Técnica donde se dan los mayores atrasos en el avance académico de los estudiantes, con la consiguiente demora en la culminación de los estudios y su incorporación a la vida laboral. Se determinó que, en las carreras técnicas o administrativas, las medias de los avances son inferiores a las carreras sociales o del área de medicina.

Al buscar relaciones entre variables socioeconómicas con el avance académico, se encontró que, para las carreras de Administración y Derecho, no se han logrado determinar variables socioeconómicas que influyan en el avance de los estudiantes. Para los estudiantes de Ingeniería Civil, el estado civil es una condición que determina el avance. Finalmente, las variables que afectan el avance en los estudiantes de Medicina son: la profesión del jefe de familia, la edad de ingreso y el tiempo desde que se gradúan del bachillerato.

Conocer algunos indicadores de las trayectorias académicas de los estudiantes: 
Gráficas de trayectorias, los avances, y la estimación de tiempo de terminación de los estudios, permite a las autoridades tomar decisiones académicas para mejorar la propuesta educativa y mejorar sus indicadores de eficiencia académica.

\section{Referencias}

Asamblea Constituyente. (2008). Constitución de la República del Ecuador. Quito, Ecuador.

Belvis, E., Moreno, M. V., y Ferrer, F. (2009). Los factores explicativos del éxito y fracaso académico en las universidades españolas, en los años del cambio hacia la convergencia europea. Revista española de educación comparada, (15), 61-92.

Bravo, F., Illescas, L., Larriva, S., y Peña, M. (2017). Causas de Deserción en el Ingreso a la Universidad; un Estudio de Caso. Revista de la Facultad de Ciencias Químicas, (18), 48-59.

Camarena, R., Chávez, A. M., y Gómez, J. (1985). Reflexiones en torno al rendimiento escolar y a la eficiencia terminal. Revista de la educación superior, (53), 1-17.

Consejo de Educación Superior. (2017). Reglamento de Régimen Académico. Quito, Ecuador.

De Garay Sánchez, A. (2004). Integración de los jóvenes en el sistema universitario: prácticas sociales, académicas y de consumo cultural. Perfiles educativos, 161-165.

Doray, P., Picard, F., Trottier, C., y Groleau, A. (2009). Educational pathways: Some key concepts. The Canada Millennium Scholarship Foundation.

Fenollar, P., Cuestas, P., y Román, S. (2008). Antecedentes del rendimiento académico: Aplicación a la docencia en Marketing. Revista Española de Investigación de
Marketing ESIC, 12(2), 7-24.

Fernández, X., y Silva, E. (2014). Deserción estudiantil universitaria en el primer semestre. El caso de una institución de educación superior ecuatoriana. Deserción, calidad y reforma universitaria. Apuntes para el debate, (10), 34-48.

Ferreyra, M.M., Avitabile, C., Álvarez, J.B., Haimovich Paz, F., y Urzúa, S. (2017). Momento decisivo: La educación superior en América Latina y el Caribe. Grupo Banco Mundial. Recuperado de: https://openknowledge.worldbank.org/bit stream/handle/10986/26489/211014ovSP .pdf

García-Castro, G., y Bartolucci, J. (2007). Aspiraciones educativas y logro académico: un estudio de caso sobre características y condiciones sociales de los estudiantes de la UAM. Revista Mexicana de Investigación Educativa, 12(35), 1267-1288

Garzón, R., Rojas, M. O., Riesgo, L. D., Pinzón, M., y Salamanca, A. L. (2010). Factores que pueden influir en el rendimiento académico de estudiantes de Bioquímica que ingresan en el programa de Medicina de la Universidad del Rosario-Colombia. Educación médica, 13(2), 85-96.

Giovagnoli, P. I. (2001). Determinantes de la deserción y graduación universitaria. Tesis de Maestría, Facultad de Ciencias Económicas, Universidad Nacional de La Plata. Recuperado de: http://sedici.unlp. edu.ar/handle/10915/37129

Illescas-Peña, L., Peña, M., Bravo, F., y Larriva, S. (2018). Metodological proposal for trajectory analysis. Case study. CEUR Workshop Proceedings, 2231, 138-147.

Kohler, J. L. (2013). Rendimiento académico, habilidades intelectuales y estrategias de aprendizaje en universitarios de Lima. 
Liberabit, 19(2), 277-288.

Labaree, R. (2013). Organizing Your Social Sciences Research Paper: Types of Research Designs. USC Libraries Research Guides. Recuperado de: https://libguides. usc.edu/writingguide

Lamas, H. A. (2015). Sobre el rendimiento escolar. Propósitos y representaciones, Revista de Psicología Educativa, 3(1), 313-386.

Lerner, J. (2011). Rendimiento académico de los estudiantes de pregrado de la Universidad EAFIT. Serie Cuadernos de Investigación. Colombia: Universidad de EAFIT.

Mares, G., Rocha, H., Rivas, O., Rueda, E., Cabrera, R., Tovar, J., y Medina, L. (2012). Identificación de factores vinculados con la deserción y la trayectoria académica de los estudiantes de Psicología en la FES Iztacala. Enseñanza e Investigación en Psicología, 17(1), 189-207.

Martínez-Padilla, J. H., y Pérez-González, J. A. (2008). Efecto de la trayectoria académica en el desempeño de estudiantes de ingeniería en evaluaciones nacionales. Formación universitaria, 1(1), 3-12.

Morales, L., Morales, V., y Holguín, S. (2016). Rendimiento escolar. Revista Electrónica de Humanidades, Tecnología y Ciencia, 15, 1-5.

Parra, C. M., Mejía, L. F., Valencia, A., Castañeda, E., Restrepo, G., Usuga, O., y Mendoza, R. (2013). Rendimiento académico de los estudiantes de primer semestre de pregrado de la Facultad de Ingeniería de la Universidad de Antioquia: cohorte 2012-2. Ingeniería y Sociedad, (6), 1-10.

Peña, M., Bravo, F., e Illescas-Peña, L. (2019). Analítica del Aprendizaje, Visualización de Trayectoria Académica. CEUR Workshop Proceedings, 2425, 11-20.
Pineda-Báez, C., Pedraza-Ortiz, A., y Moreno, I. D. (2011). Efectividad de las estrategias de retención universitaria: la función del docente. Educación y educadores, 14(1), 119-135.

Piña, J. M., y Pontón, C.B. (1997). La eficiencia terminal y su relación con la vida académica. Revista Mexicana de Investigación Educativa, 2(3), 85-102.

Rembado, F., Ramírez, S., Viera, L., Ros, M., y Wainmaier, C. (2009). Condicionantes de la trayectoria de formación en carreras científico tecnológicas: las visiones de los estudiantes. Perfiles educativos, 31(124), 8-21.

Rozo, L. J. (2008). Factores determinantes del avance académico de los estudiantes de Licenciatura en Química de la Universidad Juárez Autónoma de Tabasco (Plan Flexible). Recuperado de: http://www.rabid.ujat.mx/FilesRabidPDF /TB3752.pdf

Sepúlveda, P. G. (2013). Trayectorias académicas y construcciones subjetivas de estudiantes y ex estudiantes virtuales. Apertura: Revista De Innovación Educativa, 5(2), 70-81.

Solano Lucas, J., Frutos Balibrea, L., y Cárceles Breis, G. (2004). Hacia una metodología para el análisis de las trayectorias académicas del alumnado universitario. El caso de las carreras de ciclo largo de la Universidad de Murcia. Revista Española de Investigaciones Sociológicas (REIS), 105(1), 217-235.

Soria-Barreto, K., y Zúñiga-Jara, S. (2014). Aspectos determinantes del éxito académico de estudiantes universitarios. Formación universitaria, 7(5), 41-50.

Terigi, F. (2007). Los desafíos que plantean las trayectorias escolares. III Foro Latinoamericano de educación. Jóvenes y docentes. La escuela secundaria en el 
mundo de hoy. Fundación Santillana.

Tinto, V. (1982). Limits of theory and practice in student attrition. The Journal of Higher Education, 53(6), 687-700.

Tinto, V. (1989). Definir la deserción: una cuestión de perspectiva. Revista de la educación superior, 18(71), 33-51.

Vargas, I., Ramírez, C., Cortés, J., Farfán, A., y Heinze, G. (2011). Factores asociados al rendimiento académico en alumnos de la Facultad de Medicina: un estudio de seguimiento a un año. Salud mental, 34(4), 301-308.

Zandomeni, N., y Canale, S. (2010). Las trayectorias académicas como objeto de investigación en las instituciones de educación superior. Ciencias Económicas, 2(8), 59-65. 